\title{
La enseñanza de la historia en el aula de ELE: construcción de un PLE adaptado al profesorado poco experto
}

\author{
Próspero Manuel MoRÁN LóPEZ \\ Universidad de Oviedo -España
}

\begin{abstract}
Resumen
La diferencia entre el profesorado más jóven y el de mayor edad en la nueva era de la educación universitaria puede ser un problema para la evolución de los estudiantes que utilizan herramientas 2.0 normalmente web y un entorno de aprendizaje personal (PLE). Aquí intentamos relatar el proceso investigador y las pruebas realizadas para validar un modelo web 2.0 en un ámbito multidisciplinar en el que la aplicación sea eficaz tanto en un aula de Español como Lengua Extranjera, hacia la que se ha orientado prioritariamente el trabajo, como en estudios de humanidades, especialmente en estudios de comunicación audiovisual y de historia contemporánea, estudios donde se han realizado las prácticas descritas en este artículo. El resultado es la propuesta de un modelo de PLE (Personal Learning Enviroment o Entorno Personal de Aprendizaje) validada para su uso tanto por profesorado generacionalmente cercano a la Sociedad de la Información como por docentes de generaciones anteriores gracias a una interfaz simple e integradora que permita al alumnado trabajar en un mismo contexto en todas las asignaturas de sus estudios, llegado el caso.
\end{abstract}

Palabras clave: Organización Académica - Web 2.0 - Redes Sociales - Entornos Personales de Aprendizaje - ELE

\section{The teaching of history in the classroom of Spanish as a foreign language: Building a personal learning environment adjusted to inexpert teachers}

\begin{abstract}
The difference between older and younger professors in the new age of the university education could be a problem for the improvement of the students that uses usually web 2.0 tools and a personal learning enviroment (PLE). The paper wants to show the research and testing process to validate a web 2.0 model in multidisciplinary areas in which the application is effective both in a classroom of Spanish as a Foreign Language, to which work has focused primarily, as in humanities studies, especially in studies of visual communication and contemporary history, studies where have been conducted the practices described. The result is proposes in a model of PLE (Personal Learning Environment or Personal Learning Environment) validated for use by both, teachers generationally closest to the Information Society as teachers of previous generations, thanks to a simple and integrated interface that allows the students work in the same context in all subjects of their studies, if necessary.
\end{abstract}

Keywords: Academic Organization - Web 2.0 - Social Networks - Personal Learning Environments ELE

Referencia normalizada:

Morán López, P. M. (2013) La enseñanza de la historia en el aula de ele: construcción de un ple adaptado al profesorado poco experto. Historia y Comunicación Social. Vol. 18. № Especial Diciembre. Págs. 587-599.

Sumario: 1. Introducción. 2. Metodología y construcción de un PLE. 3. Conclusiones. 4. Bibliografía. 


\section{Introducción}

La enseñanza de humanidades se enfrenta a una importante dificultad para incorporar de forma eficiente las nuevas tecnologías en sus aulas, y con ellas los cambios socioculturales que implican, pero además lo hace en un momento en que se pone de manifiesto una importante brecha no sólo digital sino también pedagógica entre las generaciones que siguen impartiendo con métodos tradicionales las enseñanzas humanísticas y el profesorado que ha adoptado modelos constructivistas o la implementación plena en sus clases de las TIC y cuanto representan. Esta situación enfrenta al alumnado a

En la nueva era educativa en la que nos encontramos y que Weller (2011) califica como "de la abundacia", por los numerosos recursos que ponen las nuevas tecnologías a nuestra disposición, no sólo hay evidentes incompatibilidades, como las señaldas por Collins y Halverson (2010) entre los colectivos que aprenden con las nuevas tecnologías y quienes lo hacen con estilos, recursos y medios tradicionales, sino que también existen esas incompatibilidades entre el profesorado que sigue manteniéndose ajeno a las TIC y quienes las han abrazado con entusiasmo. Todo ello unido a las diferencias que marcan los modelos de aprendizaje de corte igualitario con los basados en la personalización que suponen un debate añadido. El problema que presenta la adaptación de los docentes más conservadores o de mayor veteranía a estos nuevos recursos se une la dificultad que encuentran docentes innovadores que pretenden aplicar modelos basados en estas nuevas herramientas al servicio de la didáctica y que encuentran una respuesta escasa del alumnado que se presupone adaptado a esas herramientas. $Y$ en este sentido el acercamiento a los PLE ha tenido siempre una perspectiva centrada en el discente mucho más que en el docente, tanto cuando se trataba de teorizar sobre el aprendizaje con tecnologías emergentes (Anderson, 2010) como cuando se pretendía construir un modelo de aprendizaje rizomático (Cormier, 2008) o de aprendizaje entre iguales (Corneli, 2012) culminando en la construcción de materiales docentes construidos entre iguales, online y con un modelo "open data" (Alexander, Allison, Barondeau et al., 2013).

El modelo que pretendíamos construir para enfrentarnos a reducir esa brecha digital y pedagógica tenía como referente necesario el uso de las herramientas propias de la web 2.0 pero paquetizándolas en una herramienta online que permitiera su empleo por un profesorado no experto en el uso de las TIC para su fácil aplicación en el ámbito de la educación superior de forma específicamente adaptada a asignaturas de las áreas de conocimiento mencionadas (español como lengua extranjera, comunicación audiovisual e historia contemporánea) ofreciendo en ese sentido peculiaridades específicas adaptables a los contenidos de las materias que se imparten adscritas a las mencionadas áreas de conocimiento. 


\section{Metodología y construcción de un PLE}

\subsection{El concepto de PLE y su aplicación en el aula.}

Collins y Halverson (2010) pusieron de manifiesto una cierta incompatibilidad entre el alumnado que usa en su aprendizaje de forma recurrente tecnologías y el que se aprovecha únicamente de medios tradicionales y, como consecuencia de ello, entre quienes mantienen la visión del profesor como experto único y fuente de toda la información considerada más o menos relevante frente a los que asumen sin dudart que la proliferación de fuentes de información diversas es la que prevalece actualmente no sólo en el aula sino en la vida cotidiana. En realidad estamos asistiendo al enfrentamiento entre un pasado que se resiste a fenecer, la búsqueda de fuentes únicas incontestables y plenamente confiables, con un futuro plenamente cristalizado en el presente, donde cada vez es más evidente la necesidad de emplear múltiples fuentes y de basar el aprendizaje en la capacidad para contratastar la información obtenida de esas fuentes diversas.

De hecho la enseñanza reglada ha intentado sustituir la incorporación de las TIC en el aula con múltiples fuentes recurrentes, algunas de caracter informal, por soportes tecnológicos institucionales centralizados que no constituyen sino meros remedos que se sustentantan en procesos de enseñanza-aprendizaje decididamente tradicionales (Brown, 2010).

Aquí vamos a entender el concepto de PLE, siguiendo a Adell y Castañeda (2013), como "un conjunto de herramientas, fuentes de información, conexiones y actividades que cada persona utiliza de forma asidua para aprender" teniendo en cuenta su configuración a partir de "los procesos, experiencias y estrategias que el aprendiz puede -y debe- poner en marcha para aprender" y considerando en todo caso que, "en las actuales condiciones sociales y culturales, está determinado por las posibilidades que las tecnologías abren y potencian". Por eso, entendemos que la apertura al profesorado más tradicional o conservador de opciones que le permitan incorporar, sin esfuerzos ni aprendizajes por su parte prolongados o complejos, las posiblidades que abren las TIC al aula generará numerosos beneficios tanto en los procesos de enseñanza-aprendizaje como en la coherencia de la formación propuesta de manera global tanto en enseñanzas lingüísticas como humanísiticas en el contexto del proceso de adaptación a Bolonia.

Nuestro objetivo estará en garantizar que los procesos serán coherentes con independencia del tipo de profesorado que imparta al alumnado la materia facilitando herramientas comunes:

"En consecuencia hablamos entonces de los sitios en donde re-elaboramos y publicamos la información que conseguimos: nuestro blog (en formato texto, vídeo o multimedia), un sitio de publicación de vídeos, el muro de nuestro perfil en las redes sociales, o simplemente un cuaderno de notas; pero además hablamos de los procesos que desencadenan esta reelaboración y los que la nutren -procesos de síntesis, 
reflexión, organización, estructuración, etc.-, así como de las actitudes que animan a ponerlos en marcha."(Castañeda y Adell, 2013: 17)

Consideramos aquí los PLE desde las dos perspectivas habituales en el desarrollo teórico de la propuesta. Esto es, una perspectiva teconológica referida a las aplicaciones informáticas instrumentales que son empleadas para construir el PLE de cada persona y al tiempo desde una perspectiva pedagógica propia de corrientes de pensamiento que tienen presente de manera especial al alumnado como en Attwell (2007) o Salinas (2008). Pero es la perspectiva de Lubensky (2006) definiendo el Entorno Personal de Aprendizaje como una instalación individual para acceder, agregar, configurar y manipular los artefactos digitales de las experiencias de aprendizaje la que más se acerca a nuestra propuesta por entender que esa instalación individual puede ser simultánea tanto en el plano del alumnado como en el del profesorado e imprescindible en el caso de este último colectivo para que el primero la rentabilice.

En este sentido, también resulta un referente fudamental la perspectiva de Wilson et al. (2007) de contemplar la posibilidad de construir cualquier tipo de PLE a partir de una única aplicación o de Van Harmelen (2006) que valora la creación de un PLE ad hoc. Para el desarrollo de nuestro modelo hemos tenido también en cuenta la más extendida consideración acerca de los procedimientos de implementación para un PLE (Sclater, 2008) y hemos, por tanto, construido nuestra propuesta usando tanto aplicaciones de escritorio con capacidad para trabjar offline (aunque sólo como pasarela) herramientas y servicios instalados en la nube (cloud-computing) y el propio conjunto de aplicaciones sociales más usadas en el mundo actual.

\subsection{Un modelo orientado al docente}

Al diseñar nuestra propuesta hemos tenido que centrarnos, más que en el simple uso de las plataformas digitales sobre las que se apoya, en el cambio de paradigma en la educación surgido al combinarse factores de índole social, tecnológica y económica para que la adopción práctica de un PLE en el aula no sea sólo tarea del alumnado y se limite desde la perspectiva del profesorado a una simple propuesta elaborada generalmente desde la institución, sino que se apoye en su plena integración en el formato de PLE, comprometiéndolo a la explotación del mismo y de las herramientas que integre en el trabajo cotidiano en el aula. Por eso intentamos aquí configurar una propuesta concreta de uso de las herramientas más destacadas de la Web 2.0, aplicándolas con una interface que permita su puesta en practica en un contexto interdisciplinario, pero centrado en nuestro caso en la enseñanza del español como lengua extranjera y la comunicación audiovisual y la historia, campos todos ellos interrelacionados y de presencia constante en el aula de ELE, aunque extensible a otras enseñanzas no necesariamente lingüísticas o comunicativas. El objetivo final estaría en sentar las bases técnicas y operativas de una propuesta que genere un modelo concreto de enseñanza basado en la Web 2.0 y el uso de las Redes Sociales aplicado específicamente a la enseñanza de las habilidades y técnicas que necesita un profesor de Español como Lengua Extranjera (ELE) que en el caso elegido por nosotros imparta 
historia de España a alumnos de ELE, aprovechando las posibilidades que los nuevos parámetros de la comunicación digital y las redes sociales nos ofrecen. Y que además lo haga teniendo en cuenta la trascendencia que en el aula, como reflejo de la vida cotidiana, tiene la comunicación multimedia para la obtención de cualquier objetivo en el proceso de enseñanza-aprendizaje.

La propuesta pretende, en definitiva, consolidar un modelo de software que, en combinación con un portal docente basado en Moodle ${ }^{1}$ y un sistema de blogging de las asignaturas basado en Wordpress y de redes sociales basado en Facebook y Twitter, por último, posibilite la interacción constante de los alumnos desde cualquier plataforma con un programa que facilite la conexión directa a esas y otras herramientas, pero de forma tal que el profesorado, sea cual sea su edad, formación o experiencia en las nuevas teconologías, participe de forma activa y constituya un eje central del proceso de aprendizaje en este nuevo contexto.

Concretamente, la propuesta se presenta para su desarrollo con dos submodelos perfectamente compatibles entre sí. Por un lado, con un portal basado en Moodle en el que se ofrecezca el material de una forma más cercana al e-Learning tradicional ${ }^{2}$, y por otro lado, con un conjunto de herramientas Web 2.0 que interactuarán entre sí y que se ubicarán en una plataforma desarrollada al efecto y envuelta en una interface facilitadora, para permitir su interconexión automática entre alumnado y profesorado tanto de una asignatura como de un conjunto de ellas, garantizando la modularidad del modelo.

Las herramientas elegidas para configurar este programa de trabajo han sido Wordpress como herramienta de blog, Facebook y Twitter como redes sociales, Flickr como repositorio fotográfico y You Tube como repositorio de vídeos. Todo ello, combinado con el uso de la mensajería a través de internet como forma de conexión junto con el uso sistemático del correo electrónico.

\subsection{Uso de Adobe Air en la construcción de un modelo colaborativo}

Una vez referidos algunos conceptos fundamentales para nuestro propósito, hemos diseñado un modelo colaborativo, que teniendo como eje el uso del blog, ofrezca las principales herramientas de la web 2.0, empleando para ello una plataforma, Adobe Air, que nos permite hacer de conglomerado donde unir las piezas de nuestro Puzzle.

El modelo descrito a continuación requiere de un conocimiento mínimo de las herramientas más habituales de la Web 2.0, que entendemos deberían ser conocidas en mayor o menor extensión por quienes se planteen su uso desde la perspectiva

1 La mayoría de las Universidades públicas en España vienen usando Moodle de forma mayoritaria como Campus Virtual desde hace más de un lustro una vez que la mayoría de ellas abandonaron plataformas propietarias de distintos tipos y características.

2 Obviaremos aquí descripción alguna de dicho portal basado en Moodle, por la institucionalización de este tipo de portal en la enseñanza superior española ya referido. 
docente o discente, habiendo realizado su desarrollo bajo Adobe Air y propuesto a continuación bajo el nombre de "Easywork".

Las herramientas elegidas para configurar este programa de trabajo han sido Wordpress como herramienta de blog, Facebook como red social, Flickr como repositorio fotográfico y un desarrollo propio basado en PHP Motion como repositorio de vídeos al estilo de You Tube. Todo ello, combinado con el uso de la mensajería a través de internet como forma de conexión junto con el uso sistemático del correo electrónico.

Las posibilidades de uso de nuestra aplicación EasyWork, que proporcionará acceso completo a todas las herramientas utilizadas actualmente por la web $2.0 \mathrm{y}$ recogidas en este modelo para aplicación docente, son enormes y no se circunscriben evidentemente a las areas de conocimiento descritas, sino que también puede adaptarse a muchas otras.

En primer lugar, dado que ha sido desarrollada en esa plataforma, para poder instalar nuestra aplicación debe instalarse previamente Adobe AIR desde la propia página de Adobe. $\mathrm{AIR}^{3}$ es una plataforma que permite crear aplicaciones de Internet sofisticadas que se implantan en el escritorio y se ejecutan a través de distintos sistemas operativos. Para instalar EasyWork sólo se debería ejecutar el fichero que se ofrecería a cada alumno o alumna y en un repositorio señalado por el profesorado, EASYWORK, y seguir los pasos de la instalación, pulsando la opción que ofreceríamos.

La aplicación nos da la opción de crear un acceso directo en nuestro escritorio, iniciar la aplicación una vez instalada y poder seleccionar donde deseamos que ubique la instalación de la misma. Una vez instalada la aplicación nos aparecerá, si hemos dejado las opciones por defecto, un icono en el escritorio para poder ejecutar nuestra aplicación.

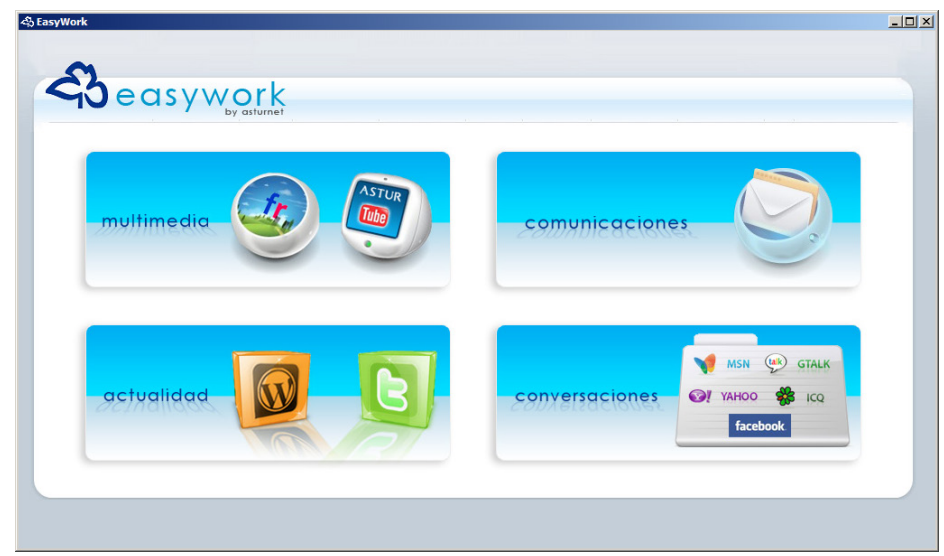

Figura 1

3 Ver http://www.adobe.com/es/products/air.html 
Como podemos observar en la ventana el interface de la aplicación Easywork está dividida en cuatro bloques o secciones que suponen también una estructura clara de la tipología de herramientas a usar. Cada una de ellas permitirá al alumnado el acceso a diferentes módulos que previamente han sido programados por el profesorado con un usuario y clave de acceso, de forma que cada alumno o alumna disponga de una interface personalizada expresamente. El acceso personalizado permitirá además al profesorado controlar (al igual que en una plataforma como Moodle) el tiempo de acceso a cada utilidad y el tiempo empleado en cada conexión o realización de una tarea concreta.

\subsection{La interface y su estructura.}

La primera sección, Multimedia, permitirá el acceso a dos módulos. Nuestro primer módulo nos va a permitir eusa una cuenta de Flickr. El profesor habrá creado una cuenta en este portal fotográfico para permitir la subida de fotografías de los alumnos al mismo. Flickr es un sitio web para compartir imágenes sobre todo fotográficas. Este popular sitio web sirve como servidor personal para compartir fotografías personales. El servicio es mundialmente usado por blogueros como un repositorio fotográfico y recientemente ha renovado su diseño y ampliado su capacidad de almacenamiento gratuito hasta un terabyte, cantidad más que suficiente para toda nuestra actividad en varias asignaturas durante un curso académico ${ }^{4}$. La popularidad en parte se debe a la gran comunidad on-line que accede al servicio, así como a las herramientas que permiten al autor etiquetar sus fotos y a un explorador de las mejores fotos de la semana. Recientemente, Flickr ha rediseñado su servicio y ha ampliado el espacio gratuito que ofrece al usuario básico hasta 1 Terabyte de almacenamiento, lo que lo convierte en una herramienta de alcance muy superior al de otras de pago de similares características 5 .

4 Vease http://www.abc.es/tecnologia/redes/20130521/abci-flickr-rediseno-cambio-201305211114. html

5 Flickr dió una gran sorpresa en este 2013 cuando decidió aumentar a 1 Terabyte la capacidad de almacenamiento ofrecida gratuitamente a sus usuarios, ya que ningún otro servicio, como 500px por ejemplo, alcanzan tal envergadura de almacenamiento. A ello ha unido en el arranque del otoño una propuesta de nuevo diseño que estará posiblemente disponible antes de finalizar 2013 y que ofrece controles más intuitivos además de una mejor experiencia de usuario. 


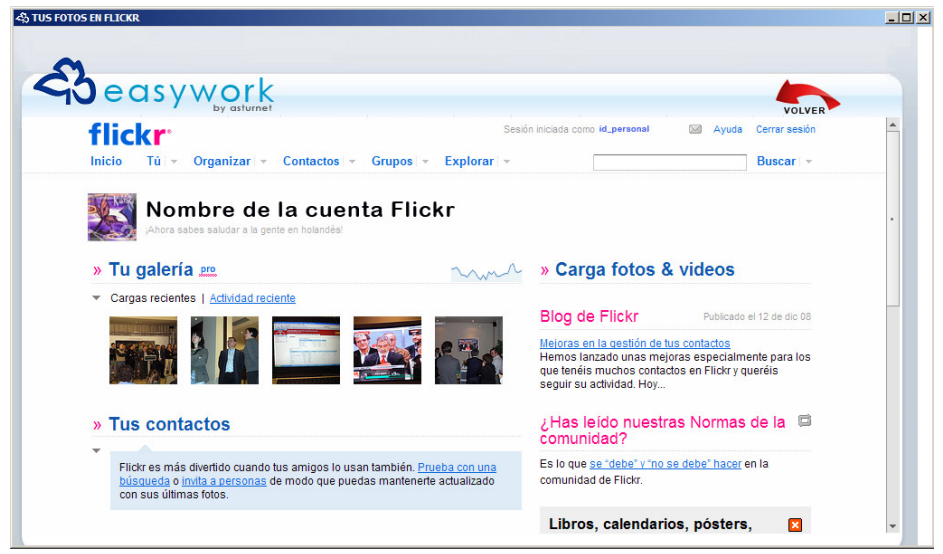

Figura 2

Una vez que pinchemos en el icono correspondiente a Flickr nos aparecerá en nuestra aplicación un acceso restringido a nuestra cuenta de Flickr. Y una vez nos hayamos validado ya tendremos acceso a todas las posibilidades que esta red social nos permite.

El segundo módulo multimedia nos va a permitir el acceso a nuestra cuenta personal en una aplicación creada bajo PHPMotion y que hemos denominado AsturTube para el ejemplo que nos ocupa, relacionado directamente con la Universidad de Oviedo, para cuyo Máster de ELE hemos creado un prototipo como ejemplificación práctica de nuestro modelo.

AsturTube sería un sitio web que permitiría a los usuarios compartir vídeos digitales a través de Internet replicando exactamente a Youtube, de forma que pudiera implementarse en su sustitución el popular portal de compartición de vídeos en la Red de redes, sobradamente conocido del alumnado universitario.

AsturTube usa, como You Tube, un reproductor en línea basado en Adobe Flash para servir su contenido, de gran eficacia gracias a la posibilidad de alojar vídeos personales de manera sencilla. La opción de personalizar nuestra aplicación de vídeos puede requerir de algunos trabajos para su personalización y optimización, por lo que resultará normalmente más eficaz para cualquier docente no avezado el uso directo YouTube, herramienta con la que se encontrará más familiarizado y que seguramente no necesitará de aprendizaje alguno.

Una nueva sección sería la de Comunicaciones que permitiría el acceso restringido y directo al webmail personal del alumno y vendría programado en la aplicación para que sea direccionado únicamente a la cuenta del profesorado al inicio del curso, bien de la propia universidad o de cualquier otro servicio.

Una tercera seccción sería la de actualidad que permitirá el acceso a dos módulos. El primero de ellos nos va a permitir el acceso al Blog de la asignatura, en nuestro caso implementado sobre WordPress por amplia extensión entre la blogosfera 
española y mundial y, sobre manera, por su facilidad de uso y sus características como gestor de contenidos. WordPress nos va a permitir manejar múltiples autores o usuarios, junto con sus roles o perfiles que establecen distintos niveles de permisos, permitiendo incluir a la totalidad del alumnado.

El segundo módulo nos va a permitir acceder de forma directa a Twitter o a un programa alternativo de micropublicación o micro-blogging completamente personalizado para la asignatura o estudio en que implementemos nuestra solución. Como en el caso del servicio para vídeos, aquí podremos optar por una solución propietaria o por el uso directo de Twitter, popular y extendido servicio de microblogging, que hace las veces de red social y que permite a sus usuarios enviar textos con una longitud máxima de 140 caracteres, que pueden incluir enlaces a imágenes y/o vídeos. Dentro de los usos más conocidos de Twitter y de las herramientas de micro-blogging en general se encuentra el seguimiento de eventos en directo, la retransmisión de charlas y ponencias a las que poca gente tiene acceso, el intercambio de opiniones durante un evento en el que la gente asiste como público o incluso comentarios sobre películas o debates retransmitidos por la televisión ${ }^{6}$.

La intención de esta presencia de Twitter en el paquete de aplicaciones que aquí proponemos para su desarrollo como plataforma es que la totalidad del alumnado pueda seguir la actividad de sus compañeros y compañeras, bien para facilitar la comunicación con los que formen grupos de trabajo eventuales o fijos durante el desarrollo temporal del proceso de enseñanza-aprendizaje de que se traye o bien para facilitar la comunicación personal entre ellos o entre ellos y el profesorado.

En nuestra propuesta inicial la conexión no se realizaría a Twitter (twitter.com) sino a un servidor que emularía las características de Twitter y un servicio expresamente creado para la ocasión, a partir de la aplicación de libre distribución (Open Source) MiniTwitter. De esta forma el mencionado Minitwitter tendría únicamente como usuarios a nuestro alumnado y estaría operativo únicamente durante el curso académico, pudiendo complementarse con una cuenta personal del alumno en Twitter sin ningún problema, ni interferencia. No obstante, también podría implementarse directamente el uso de Twitter directamente en la aplicación para cohesionar y racionalizar la actividad.

Finalmente, la sección de comunicaciones nos permitiría el acceso a las principales redes sociales y a los sistemas de mensajería, tan útiles para la tutorización a distancia y tan empleados por los jóvenes en España. La aplicación permitiría las opciones más habituales en nuestro país. En la actualidad el uso del Gtalk del Google o del chat de Facebook han vencido en este campo a clásicos como el Messenger de Microsoft o el pionero ICQ o el servicio de Yahoo, pudiendo ser empleados todos simultáneamente o pudiendo ser seleccionado tan sólo alguno que consideremos más eficaz, bien por su mayor aceptación, empleo o conocimiento entre el alumnado.

6 El ejemplo habitual en nuestro país como arranque del modelo es el debate entre Zapatero y Rajoy durante las elecciones presidenciales de España 2008, pero en la actualidad son innumerables las referencias que podrían traerse a colación. 
Esta sección nos permitirá el acceso directo a través de nuestra aplicación a todos los servicios que Google nos ofrece desde su aplicación GTalk. La sección pretende servinos también de pasarela al acceso directo a una página o grupo de Facebook que hubieran sido creados por cada profesor para su asignatura y además al perfil propio del alumno si lo tuviera. El grupo funciona como una especie de grupo de discusión compartido para interactuar sobre la materia de la asignatura, aprovechando las opciones y servicios que Facebook ofrece a través de la web.

\subsection{La web como punto de entrada .}

El complemento a "Easywork" como interface para la interacción entre alumnado y profesorado con las herramientas web 2.0 seleccionadas será, junto con el portal basado en Moodle de carácter institucional que pone la Universidad a nuestra disposición, una alternativa web a ese portal institucional que nosotros hemos desarrollado. El portal diseñado se estructura de forma modular, permitiendo acceder a las mismas referencias que la interface "Easywork" ya descrita, pero añadiendo además algunas herramientas que no son necesarias para la interacción del profesorado (como la red social Tuenti mayoritaria aún entre el alumnado español recién llegado a la Universidad pero de escasa implantación entre adultos) y que el profesorado puede construir a través de un simple formulario que se encarga bajo programación en PHP de crear un portal de características similares al mostrado en la figura 3 simplemente seleccionando de nustro ordenador una imagen e introduciendo datos de nuestra cuenta en Google, Yahoo o cualquier otro servicio de similares características que usaríamos en la interface ya descrita con anterioridad.

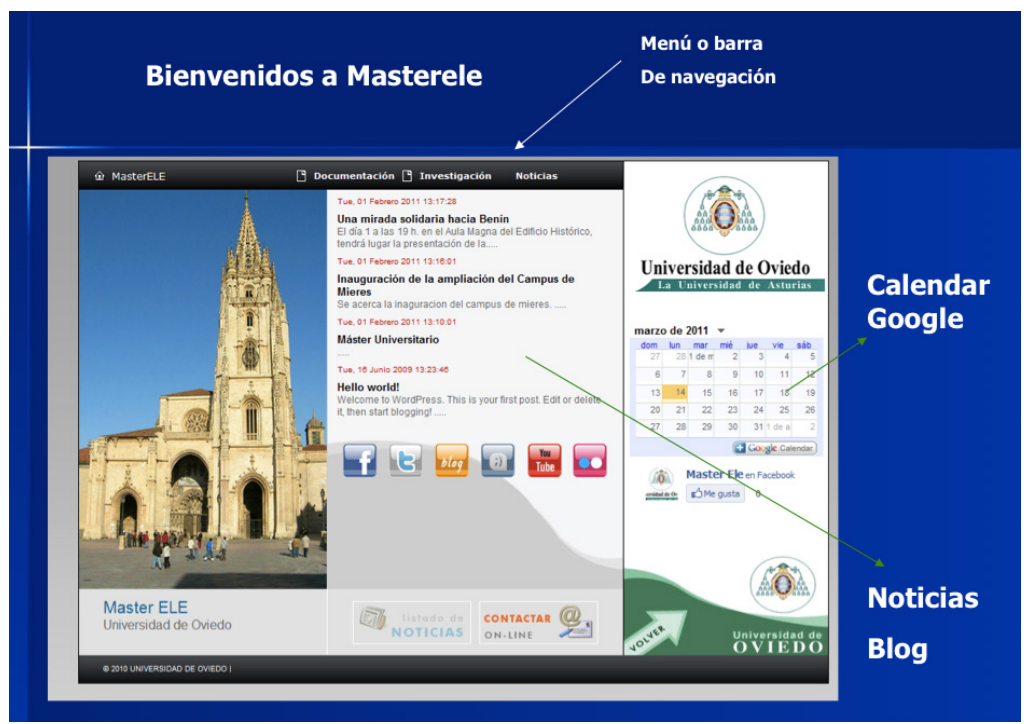

Figura 3 


\section{Conclusiones}

La implementación de la interface "Easywork" y del portal de inicio referido ha sido realizada con destino a un Máster Oficial en ELE de la Universidad de Oviedo y con la vista puesta en la impartición dentro de ese Máster de una asignatura de Historia de España, fiel reflejo de la que habitualmente se imparte como complemento contextualizador a la enseñanza estrictamente lingüística en los cursos de española para extranjeros en nuestro país. Pero su diseño modular ha previsto la posibilidad de ser adaptada sin dificultad y de forma directa a cualquier asignatura o curso lingüístico, haciéndolo igualmente extensible a la mayoría de las asignaturas de humanidades o del ámbito de la comunicación periodística o audiovisual. Y de esa implementación hemos extraído tres conclusiones fundamentales:

1. La importancia evidente de los sistemas de E-learning en el tratamiento didáctico de una disciplina humanística, como ocurre con la enseñanza del español como lengua extranjera, que necesita de una visualización mayor, o de la enseñanza de la historia, en ese mismo contexto introductorio o en el contexto especializado de un Master en ELE.

2. La potencialidad de los planteamientos expuestos por la cercanía del previsible alumnado de ELE al mundo de las redes sociales en particular y de las TIC en general.

3. La superación, gracias al modelo implementado, de las dificultades habituales que presenta la aplicación de este tipo de propuestas ante la ausencia de una oferta formativa amplia para el profesorado en el contexto de las TIC, precedida de una necesaria concienciación de entre el profesorado de más avanzada edad al que afecta de forma directa la brecha digital.

\section{Bibliografía}

ALEXANDER, B., ALLISON, P., BARONDEAU, R., et al., (2012). The Peeragogy Handbook. Online (martes 1 de enero de 2013, version 1). Disponible en http:// peeragogy.net/peeragogy-handbook-v1.pdf. Consultado el 8 de enero de 2013.

ATTWELL, G. (2007). Personal learning environments-the future of elearning? eLearning Papers, 2(1), 1-7. Disponible en http://www.elearningeuropa.info/files/ media/media11561.pdf . Consultado el 9 de enero de 2013.

BROWN, S. (2010). From VLEs to learning webs: the implications of Web 2.0 for learning and teaching. Interactive Learning Environments 18(1), 1-10.

CARDOSO, Gustavo (2008) Los medios de comunicación en la Sociedad Red. Filtros, escaparates y noticias. Barcelona: UOC.

CEREZO J.M. (coord.) (2006) La blogosfera hispana: pioneros de la Cultura Digital, Madrid: Fundación France Telecom. 
COLLINS, A.; HALVERSON, R. (2010). The second educational revolution: Rethinking education in the age of technology. Journal of Computer Assisted Learning, 26(1), 18-27. doi:10.1111/j.1365-2729.2009.00339.

CORMIER, D. (2008). Rhizomatic education: Community as curriculum. Innovate: Journal of Online Education, 4(5). Disponible en http://www.innovateonline. info/pdf/vol4_issue5/Rhizomatic_Education_Community_as_Curriculum.pdf . Consultado el 8 de enero de 2013 .

CORNELI, J. (2012) Paragogical Praxis, E-Learning and Digital Media, 9(3), 267-272. Disponible en http://www.wwwords.co.uk/rss/abstract.asp?j=eleayaid $=509$. Consultado el 9 de enero de 2013.

LUBENSKY, R. (2006) The present and future of Personal Learning Environments (PLE). Disponible en http://members.optusnet.com.au/rlubensky/2006/12/ present-and-future-ofpersonal-learning.html. Consultado el 8 de enero de 2013.

MOMINÓ, J. Ma; SIGALÉS, C.; MENESES, J. (2008) La escuela en la sociedad red. Internet en la educación primaria y secundaria. Barcelona: Ariel.

SALINAS, J. (2008). Algunas perspectivas de los entornos personales de aprendizaje. TICEMUR Jornadas Nacionales de TIC en la educación. Lorca, Murcia. Disponible en http://gte.uib.es/pape/gte/publicaciones/algunas-perspectivas-de-los-entornos-personales-de-aprendizaje. Consultado el 8 de enero de 2013.

SCLATER, N. (2008). Web 2.0, personal learning environments, and the future of learning management systems. ECAR, 2008(13).

VAN HARMELEN, M. (2006). Personal learning environments. In R. Kinshuk, P. Koper, P. Kommers, D. Kirschner, W. Didderen, y Sampson (Eds.), Proceedings of the Sixth International Conference on Advanced Learning Technologies (pp. 815-816). Los Alamitos, CA: IEEE Computer Society.

WELLER, M. (2011). A pedagogy of abundance. Revista Española de Pedagogía, 249, 223-236

WENGER, E. C., McDERMOTT, R., AND SNYDER, W. C. (2002), Cultivating Communities of Practice: A Guide to Managing Knowledge, USA: Harvard Business School Press, Cambridge.

WILSON, S., LIBER, O., JOHNSON, M., BEAUVOIR, P., SHARPLES, P. y MILLIGAN, C. (2007). Personal learning environments: Challenging the dominant design of educational systems. Journal of e-Learning and Knowledge Society, $3(2), 27-38$.

Capítulos o artículos en libros o revistas en papel

AGUADED-GÓMEZ, J.I. (1999). Educar para los medios es educar para la democracia. Comunicar (Madrid) 13; 6-8.

CASTAÑEDA, L; ADELL, J. (Eds.) (2013). Entornos Personales de Aprendizaje: claves para el ecosistema educativo en Red. Alcoy: Marfil.

CANTALAPIEDRA, M.J. Y MORÁN LÓPEZ P.M., (2006) Nuevas expresiones, nuevos públicos en DÍAZ-NOSTY, B., TENDENCIAS 06/MEDIOS DE COMUNICACIÓN, (381-394), Madrid: Fundación Telefónica. 
COLL, César (2007) Las competencias en la educación escolar: Algo más que una moda y mucho menos que un remedio. Aula de innovación educativa, 161, (34-39).

COLLINS, A y HALVERSON, R. (2010). The second educational revolution: rethinking education in the age of technology. Journal of Computer Assisted Learning, 26: $18-27$

GABELAS, A. (2007) Una perspectiva de la educación en medios para la comunciacion en España. Comunicar, 28 (Madrid), 69-73.

WELLER, Martin (2011). A pedagogy of abundance. Spanish Journal of Pedagogy, 249 pp. $223-236$

LÓPEZ ARES, SUSANA y MORÁN LÓPEZ, P.M. (2008) Usos del blog como apoyo docente en la programación de cursos de verano. En Libro de Actas de las II Jornadas de Intercambio de Experiencias en Docencia Universitaria en la Universidad de Oviedo. Vicerrectorado de Actividad Académica, Profesorado y Convergencia Europea. Universidad de Oviedo. Oviedo (341-349)

MORÁN LÓPEZ, P.M. (2004) "Nuevos modelos para un aprovechamiento didáctico del periodismo digital", en Estudios de Periodística XI: El periodismo motor de paz, ( pg. 321-334). Madrid: Sociedad Española de Periodística.

Artículos en publicaciones web

BALLANO MACÍAS, S. (2010., El papel de los medios de comunicación y las TIC en la Educación Formal. Aportaciones para la concreción de la noción de alfabetización mediática. Disponible en: http://www.aeic2010malaga.org/upload/ ok/297.pdf. Consultado el 22 de julio de 2013.

BARTOLOMÉ, A. (2008). "Web 2.0 and New Learning Paradigms". e-Learning Papers. Disponible en: http://www.e-Learningpapers.eu/index.php?page=doc\&doc $\_$id $=11654 \&$ doclng $=6 \& \mathrm{~V}$ ol $=8$.

COBB, J. (2008). Learning 2.0 for Associations. Mission to Learn blog. Disponible en: http://blog.missiontolearn.com/2008/02/learning-20-ebook-free/ Consultado el 22 de julio de 2013.

DOWNES, S. (2007). E-Learning 2.0 in Development. Disponible en: http://www. slideshare.net/Downes/e-Learning-20-in-development. Consultado el 24 de julio de 2013

DOWNES, S. E-Learning 2.0. eLearn Magazine. Disponible en: http://www.elearnmag.org/subpage.cfm?section=articles\&article $=29-1$ Consultado el 22 de julio de 2013.

MORÁN LÓPEZ, P.M.(2003) Una aproximación al weblog. Ponencia presentada al III Congreso de Periodismo Digital, Huesca. Disponible en: http://www.diariored. $\mathrm{com} / \mathrm{blog} / \mathrm{ana} / \mathrm{archivo} / 000706 . p h p$ Consutado el 10 de julio de 2010.

SIEMENS, G. (2008). Learning and Knowing in Networks: Changing roles for Educators and Designers: Teacher as Network Administrator. http://it.coe.uga. edu/itforum/Paper105/Siemens.pdf . Consutado el 10 de julio de 2010.

VV.AA., Mission to learn. Learning 2.0: A resource Guide (2007). Disponible en: http://www.missiontolearn.com/resources/M2L_Learning20_resources.pdf Consultado el 22 de julio de 2013 